\title{
Correlation of Videofluoroscopic Swallowing Study Findings With Radionuclide Salivagram in Chronic Brain-Injured Patients
}

\author{
Ga Yang Shim, MD, Ju Sun Oh, MD, Seunghee Han, MD, \\ Kyungyeul Choi, MD, Son Mi Lee, MD, Min Woo Kim, MD
}

Department of Physical Medicine and Rehabilitation, Seoul Medical Center, Seoul, Korea

Objective To investigate the correlation between videofluoroscopic swallowing study (VFSS) and radionuclide salivagram findings in chronic brain-injured patients with dysphagia.

Methods Medical records of chronic brain-injured patients who underwent radionuclide salivagram and VFSS were retrospectively analyzed. Patients were divided into two groups according to salivagram findings. Differences in patient characteristics and clinical factors, including Mini-Mental State Examination (MMSE), Modified Barthel Index (MBI), Functional Ambulatory Category (FAC), feeding method, tracheostomy state, and VFSS findings between the two groups were investigated.

Results A total of 124 patients were included in this study. There were no significant differences in MMSE, MBI, FAC, feeding method, and presence of tracheostomy between the two groups. However, the incidence of aspiration pneumonia history was significantly higher in the positive salivagram group. The Functional Dysphagia Scale (FDS) was significantly associated with positive salivagram findings, especially in the pharyngeal phase. A multivariate logistic regression analysis showed that laryngeal elevation and epiglottic closure was statistically significant FDS parameter in predicting salivary aspiration on a salivagram (odds ratio=1.100; 95\% confidence interval, 1.017 1.190; $\mathrm{p}=0.018$ ). The receiver operating characteristic (ROC) curve of FDS in the pharyngeal phase showed that an optimum sensitivity and specificity of $55.1 \%$ and $65.4 \%$, respectively, when the cut-off value was 39 .

Conclusion In chronic brain-injured patients, inappropriate laryngeal elevation and epiglottic closure is predictive variable for salivary aspiration. Therefore, performing a radionuclide salivagram in patients with FDS of 39 or less in the pharyngeal phase for prevents aspiration pneumonia from salivary aspiration.

Keywords Radionuclide salivagram, Videofluoroscopic swallowing study, Aspiration pneumonia, Deglutition

Received July 30, 2020; Revised September 29, 2020; Accepted October 14, 2020; Published online April 21, 2021

Corresponding author: Ju Sun Oh

Department of Physical Medicine and Rehabilitation, Seoul Medical Center, 156 Sinnae-ro, Jungnang-gu, Seoul 02053, Korea. Tel: +82-2-2276-7474, Fax: +82-2-2276-7477, E-mail: sunnywind78@gmail.com

ORCID: Ga Yang Shim (https://orcid.org/0000-0002-0475-311X); Ju Sun Oh (https://orcid.org/0000-0002-0994-3051); Seunghee Han (https://orcid. org/0000-0002-0273-3609); Kyungyeul Choi (https://orcid.org/0000-0003-4218-0102); Son Mi Lee (https://orcid.org/0000-0001-5014-4107); Min Woo Kim (https://orcid.org/0000-0003-3100-9692).

(a) This is an open-access article distributed under the terms of the Creative Commons Attribution Non-Commercial License (http://creativecommons.org/ licenses/by-nc/4.0) which permits unrestricted noncommercial use, distribution, and reproduction in any medium, provided the original work is properly cited. Copyright $\odot 2021$ by Korean Academy of Rehabilitation Medicine 


\section{INTRODUCTION}

Dysphagia is a common complication that may cause malnutrition and aspiration pneumonia in patients with brain injury [1]. Aspiration pneumonia is the major cause of death, especially in patients with chronic brain lesion. Aspiration occurs as a result of swallowing dysfunction, gastroesophageal reflux, or inability to adequately protect the airway from oral secretions [2]. Aspiration pneumonia is frequently seen in patients with long-term tube feeding, suggesting it results from salivary aspiration in patients with chronic brain-injured patients [3]. Therefore, it is important to detect salivary aspiration for preventing pneumonia.

Videofluoroscopic swallowing study (VFSS) is the gold standard for evaluating swallowing difficulties [4]. VFSS can visualize the oropharyngeal and esophageal phases of swallowing and provide detailed information on the anatomy and physiology of swallowing [2]. However, it is challenging to diagnose aspiration pneumonia in chronic brain-injured patients because salivary aspiration is the least recognized form of aspiration and episodic phenomenon that cannot be detected by VFSS [5-8].

A radionuclide salivagram is a useful tool for detecting salivary aspiration. It uses the scintigraphic method and traces material in the respiratory tract or not. It is used mostly in children with cerebral palsy to evaluate swallowing difficulties as it is safe and does not require radiation exposure or adequate cooperation $[9,10]$. Although the salivagram is a sensitive test as reported in previous studies, it is not established as a formal test for detecting salivary aspiration [10-12].

Several studies have compared VFSS and salivagram. However, most have focused on children or heterogeneous disease groups. In addition, studies on chronic brain-injured patients with salivary aspiration are limited although these patients are commonly seen in a rehabilitation unit. Therefore, the aim of this study is to investigate the correlation between VFSS and radionuclide salivagram in chronic brain-injured patients with dysphagia.

\section{MATERIALS AND METHODS}

\section{Subjects}

This was a cross-sectional retrospective study of chronic brain-injured patients who had radionuclide salivagram and VFSS for detecting salivary aspiration from January 2013 to February 2020 at Seoul Medical Center rehabilitation unit. Characteristics of patients, findings of VFSS and salivagram and clinical factors were obtained from their medical records. The inclusion criteria were (1) adult patients (>18 years old) who had brain injury, (2) those whose disease duration was at least 3 months since brain injury. Exclusion criteria were (1) a previous history of brain lesion, (2) any underlying disease that could affect swallowing function, such as neurodegenerative disease (idiopathic Parkinson disease, Alzheimer dementia), brain tumors and epilepsy, (3) patients with more than 1-week interval between the salivagram and VFSS, and (4) those with insufficient medical records. This study was approved by the Institutional Review Board of Seoul Medical Center (No. 2020-04-036). Written informed consents were obtained.

\section{Patients' characteristics and clinical factors}

To evaluate the correlation between VFSS and salivagram findings, patient characteristics such as age, sex, disease duration, types of impairment and lesion location were analyzed. Additionally, clinical factors such as Mini-Mental State Examination (MMSE), the Modified Barthel Index (MBI), the Functional Ambulatory Category (FAC), feeding method, the presence of tracheostomy were analyzed. History of pneumonia was investigated through a retrospective review of charts from 1 month before and after VFSS and salivagram. The criteria were the presence of symptoms (fever, cough or sputum), associated radiological evidence of aspiration pneumonia and use of antibiotics.

\section{Videofluoroscopic swallowing study}

VFSS was performed in a fluoroscopy room with the patients seated in an upright position. Dynamic fluoroscopic images were acquired with lateral and anteriorposterior views. Swallowed materials were comprised water, yogurt, boiled rice, and cookies mixed with barium sulfate (Solotop Suspension; Taejoon Pharm Co. Ltd., Seoul, Korea). The results were analyzed by two experienced rehabilitation medicine physicians.

Penetration-Aspiration Scale (PAS), an 8-point numerical scale, was used for qualitative evaluation of penetration or aspiration during VFSS [13]. In this study, negative 
aspiration was defined as a PAS of 1 to 5 while positive aspiration was greater than $5[9,14]$ (Supplementary Table S1).

Functional Dysphagia Scale (FDS), a numerical scale, was utilized for the quantitative evaluation of dysphagia severity (from 0 to 100) [15], with a high score indicating poor oropharyngeal dysfunction. FDS has 11 parameters to indicate the swallowing function of the oral and pharyngeal phase. These parameters include lip closure, bolus formation, residue in oral cavity, oral transit time, triggering of pharyngeal swallowing, laryngeal elevation and epiglottic closure, nasal penetration, vallecular sinus residue, pyriform sinus residue, coating of the pharyngeal wall and pharyngeal transit time. All parameters were quantified using $3 \mathrm{~mL}$ thin liquid of diluted barium (Supplementary Table S2).

\section{Radionuclide salivagram}

Radionuclide salivagram was used to detect salivary aspiration by assessing radioactivity in trachea or not. One milliliter of diluted ${ }^{99 \mathrm{~m}}$ Tc-DTPA (technetium-99m labeled diethylenetriaminepentaacetic acid) was administrated under the tongue of patient in a supine position. Dynamic acquisition for posterior images was conducted for the first 10 minutes, followed by a delayed static acquisition for with anterior and posterior images at 0,10 , and 30 minutes. Images were taken by gamma camera (BrightView XCT Imaging System; Philips, Cleveland, $\mathrm{OH}, \mathrm{USA})$. These images were analyzed by experienced nuclear medicine physicians. Aspiration was defined as detectable radioactivity within the tracheobronchial tree.

\section{Statistical analysis}

All statistical analyses were performed using SPSS version 23.0 (IBM SPSS, Armonk, NY, USA). For comparison between groups, a Mann-Whitney test and Fisher exact test or Pearson chi-square test was used. A multivariate logistic regression analysis with forward stepwise selection was performed to identify statistically significant variables associated with aspiration of radionuclide salivagram. A receiver operating characteristic (ROC) curve was used to determine the reference value showing optimal sensitivity and specificity. A p-value less than 0.05 was considered statistically significant.

\section{RESULTS}

\section{Patient characteristics}

After screening 182 chronic brain-injured patients with dysphagia, a total of 124 patients were finally included. The patients were divided into two groups according to the presence of salivary aspiration on a salivagram. Sixtynine patients $(55.6 \%)$ were positive for salivary aspiration while 55 (44.4\%) were negative. Patient characteristics are presented in Table 1. There were no significant differences in age, sex, disease duration, types of impairment and lesion location between two groups. Regarding functional state, there was no significant difference in MMSE, FAC,

Table 1. Patients' characteristics according to salivagram findings $(n=124)$

\begin{tabular}{|lccc}
\hline \multicolumn{1}{|c}{ Demographic factors } & Salivagram aspiration $(+)(\mathbf{n = 6 9 )}$ & Salivagram aspiration (-) (n=55) & p-value \\
\hline Age $(\mathrm{yr})$ & $60.68 \pm 13.175$ & $56.85 \pm 18.383$ & 0.388 \\
\hline Sex & & & 0.785 \\
\hline Male & $48(69.6)$ & $37(67.3)$ & 0.211 \\
\hline Female & $21(30.4)$ & $18(32.7)$ & \\
\hline Types of impairment & & & $16(29.1)$ \\
\hline Ischemic stroke & $10(14.5)$ & $24(43.6)$ & 0.653 \\
\hline Hemorrhagic stroke & $41(59.4)$ & $14(25.5)$ & $1(1.8)$ \\
\hline Traumatic brain injury & $14(20.3)$ & & \\
\hline Hypoxic brain injury & $4(5.8)$ & $18(32.7)$ & 0.541 \\
\hline Location of brain lesion & $20(29.0)$ & $37(67.3)$ & $14.53 \pm 13.354$ \\
\hline Unilateral lesion & $49(71.0)$ & & \\
\hline Bilateral lesion & $12.65 \pm 9.508$ & & \\
\hline Disease duration $(m o)$ & & & \\
\hline
\end{tabular}

Values are presented as mean \pm standard deviation or number (\%). 
Table 2. Clinical factors according to salivagram findings

\begin{tabular}{|c|c|c|c|c|}
\hline Variable & Salivagram aspiration $(+)(n=69)$ & Salivagram aspiration $(-)(n=55)$ & Total $(n=124)$ & p-value \\
\hline MMSE & $6.86 \pm 8.247$ & $7.71 \pm 10.210$ & $7.23 \pm 9.140$ & 0.701 \\
\hline MBI & $9.90 \pm 14.698$ & $11.24 \pm 14.867$ & $10.49 \pm 14.728$ & 0.834 \\
\hline FAC & $0.54 \pm 0.833$ & $0.58 \pm 1.100$ & $0.56 \pm 0.957$ & 0.457 \\
\hline Feeding method & & & & 0.060 \\
\hline Tube feeding & $66(95.7)$ & $47(85.5)$ & $113(91.1)$ & \\
\hline Oral feeding & $3(4.3)$ & $8(14.5)$ & $11(8.9)$ & \\
\hline Tracheostomy & & & & 0.190 \\
\hline Tracheostomy (+) & $58(84.1)$ & $41(74.5)$ & $99(79.8)$ & \\
\hline Tracheostomy (-) & $11(15.9)$ & $14(25.5)$ & $25(20.2)$ & \\
\hline Pneumonia history & & & & $0.013^{*}$ \\
\hline $\mathrm{AP}(+)$ & $43(62.3)$ & $22(40.0)$ & $65(52.4)$ & \\
\hline $\mathrm{AP}(-)$ & $26(37.7)$ & $33(60.0)$ & $59(47.6)$ & \\
\hline
\end{tabular}

Values are presented as mean \pm standard deviation or number (\%).

MMSE, Mini-Mental State Examination; MBI, Modified Barthel Index; FAC, Functional Ambulatory Category; AP, aspiration pneumonia.

${ }^{*} \mathrm{p}<0.05$, statistically significant in Pearson chi-square test.

Table 3. Correlations of VFSS with radionuclide salivagram

\begin{tabular}{cccc}
\hline & Salivagram aspiration $(+)(\mathbf{n}=69)$ & Salivagram aspiration $(-)(\mathbf{n}=\mathbf{5 5})$ & p-value \\
\hline VFSS & & & $0.011^{*}$ \\
aspiration (+) & $47(68.1)$ & $25(45.5)$ & \\
aspiration (-) & $22(31.9)$ & $30(54.5)$ & \\
\hline
\end{tabular}

Values are presented as mean \pm standard deviation or number (\%).

A total agreement is $62 \%$ and kappa is 0.228 .

VFSS, videofluoroscopic swallowing study.

${ }^{*} \mathrm{p}<0.05$, statistically significant in Pearson chi-square test.

and MBI score either between the two groups (Table 2). Other clinical factors such as feeding method and presence of tracheotomy showed no significant difference between the two groups. However, the incidence of aspiration pneumonia was significantly higher in the positive group than in the negative salivagram group $(\mathrm{p}=0.013)$.

\section{Dysphagia severity and characteristics}

Dysphagia characteristics according to salivagram findings are summarized in Tables 3 and 4. There was statistical association between VFSS and salivagram ( $\mathrm{p}=0.011)$. The total agreement between the two tests was $62 \%$ and the kappa value was 0.228 . Statistically significant differences were observed for PAS as a qualitative evaluation (5.74 \pm 2.842 in positive finding vs. $4.11 \pm 3.071$ in negative finding; $p=0.004$ ) and total FDS score indicating the severity of dysphagia $(52.96 \pm 15.971$ in positive finding vs.
44.45 \pm 19.433 in negative finding; $\mathrm{p}=0.013$ ). There was a significant difference in FDS parameters such as triggering of pharyngeal swallowing $(\mathrm{p}=0.009)$, laryngeal elevation and epiglottic closure $(\mathrm{p}=0.008)$ and nasal penetration $(\mathrm{p}=0.026)$ between the two groups. The multivariate logistic regression analysis with the forward stepwise method demonstrated that laryngeal elevation and epiglottic closure in FDS parameters was significantly associated with positive findings on the salivagram (odds ratio $=1.100$; 95\% confidence interval [CI], 1.017-1.190; $\mathrm{p}=0.018$ ) (Table 5). In the ROC curve analysis, the area under the ROC curve (AUC) of total FDS and FDS in the pharyngeal phase for salivary aspiration on salivagram was 0.686 (95\% CI, $0.530-0.730 ; \mathrm{p}<0.013$ ) and $0.630(95 \%$ CI, 0.592-0.779; p<0.0001), respectively (Fig. 1). The optimal cut-off value for total FDS was 50.5 (sensitivity $=56.5 \%$, specificity $=61.8 \%$ ) and for FDS in the pharyn- 
Table 4. Swallowing characteristics and severity of dysphagia according to salivagram findings

\begin{tabular}{|lccc}
\hline \multicolumn{1}{c}{ Swallowing characteristic } & $\begin{array}{c}\text { Salivagram } \\
\text { aspiration }(+)(\mathbf{n}=\mathbf{6 9})\end{array}$ & $\begin{array}{c}\text { Salivagram } \\
\text { aspiration }(-)(\mathbf{n}=\mathbf{5 5})\end{array}$ & p-value \\
\hline Penetration-Aspiration Scale & $5.74 \pm 2.842$ & $4.11 \pm 3.071$ & $0.004^{*}$ \\
Functional Dysphagia Scale & $52.96 \pm 15.971$ & $44.45 \pm 19.433$ & $0.013^{*}$ \\
\hline Oral phase & & & \\
$\quad$ Lip closure & $3.48 \pm 4.050$ & $4.00 \pm 4.850$ & 0.789 \\
\hline Bolus formation & $2.87 \pm 1.580$ & $2.67 \pm 1.796$ & 0.495 \\
\hline Residue in oral cavity (\%) & $2.35 \pm 2.057$ & $2.69 \pm 2.252$ & 0.439 \\
\hline Oral transit time (s) & $3.65 \pm 2.950$ & $3.71 \pm 2.942$ & 0.915 \\
\hline Pharyngeal phase & & & \\
\hline Triggering of pharyngeal swallowing & $7.83 \pm 4.155$ & $5.82 \pm 4.978$ & $0.009^{*}$ \\
\hline Laryngeal elevation and epiglottic closure & $10.61 \pm 3.870$ & $8.73 \pm 5.394$ & $0.008^{*}$ \\
\hline Nasal penetration (\%) & $0.75 \pm 1.850$ & $0.15 \pm 0.756$ & $0.026^{*}$ \\
\hline Residue in the valleculae (\%) & $6.03 \pm 3.670$ & $4.95 \pm 3.352$ & 0.095 \\
\hline Residue in the pyriform sinuses (\%) & $6.14 \pm 3.731$ & $4.80 \pm 4.314$ & 0.052 \\
\hline Coating of pharyngeal wall after swallowing & $7.39 \pm 4.423$ & $6.36 \pm 4.855$ & 0.219 \\
\hline Pharyngeal transit time (s) & $1.86 \pm 2.009$ & $1.45 \pm 1.942$ & 0.264 \\
\hline
\end{tabular}

Values are presented as number (\%) or mean \pm standard deviation.

${ }^{*} \mathrm{p}<0.05$, statistically significant in Mann-Whitney test.

Table 5. Multivariate logistic regression analysis of variables associated with salivary aspiration on salivagram

\begin{tabular}{lcccc}
\multicolumn{1}{c}{ Parameter } & OR & $\mathbf{9 5 \%}$ CI & $\boldsymbol{\beta}$ & p-value \\
\hline Laryngeal elevation and epiglottic closure & 1.100 & $1.017-1.190$ & 0.095 & $0.018^{*}$ \\
Nasal penetration & 1.426 & $0.980-2.075$ & 0.355 & 0.064 \\
\hline
\end{tabular}

OR, odds ratio; $\mathrm{CI}$, confidence interval; $\beta$, standardized estimates.

${ }^{*} \mathrm{p}<0.05$, statistically significant in multivariate logistic regression analysis with forward stepwise method.

(A)

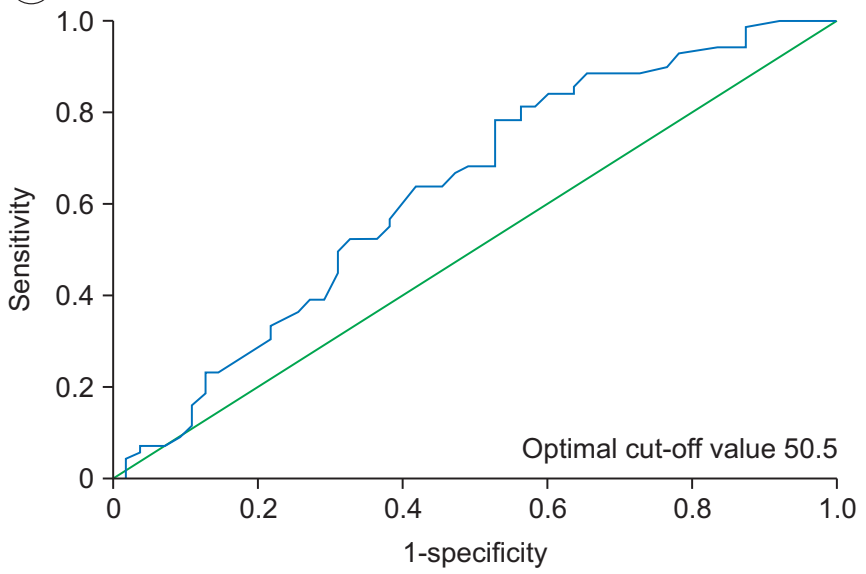

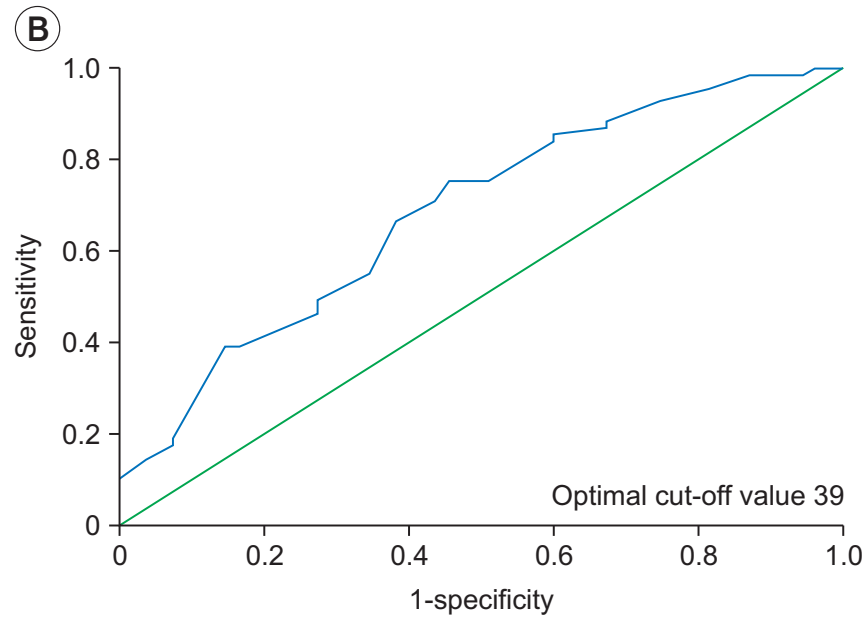

Fig. 1. (A) ROC curve of total FDS for salivary aspiration on salivagram. The optimal cut-off value is 50.5 (AUC=0.630; 95\% CI, 0.530-0.730; $\mathrm{p}=0.013$; sensitivity $=56.5 \%$, specificity=61.8\%). (B) ROC curve of FDS in pharyngeal phase for salivary aspiration on salivagram. The optimal cut-off value is 39 (AUC=0.686; 95\% CI, 0.592-0.779; $<<0.0001$; sensitivity $=55.1 \%$, specificity=65.4\%). ROC, receiver operating characteristic; FDS, Functional Dysphagia Scale; AUC, area under the ROC curve; CI, confidence interval. 
geal phase was 39 (sensitivity=55.1\%, specificity=65.4\%).

\section{DISCUSSION}

This study investigated the correlation between VFSS and salivagram findings in patients with chronic brain injury. There was no significant difference in patient characteristics or clinical factors between patients with positive findings of salivary aspiration on salivagram and those with negative findings. However, statistically significant differences were found in the swallowing functions, especially in airway protective mechanisms such as triggering of pharyngeal swallowing, laryngeal elevation and epiglottic closure and nasal penetration. According to the multivariate logistic regression analysis, laryngeal elevation and epiglottic closure of the FDS parameter was associated with salivary aspiration. Therefore, appropriate airway protective mechanisms are important as they are related to the occurrence of aspiration pneumonia in patients with chronic brain injury for preventing salivary aspiration.

VFSS and radionuclide salivagram are useful tools for detecting aspiration. In the present study, the total agreement was $62 \%$ and the kappa value was 0.228 , although there was a statistical association between the two tests $(\mathrm{p}=0.011)$. The consistency was lower than expected, which is consistent with previous studies $[9,10]$. Possible explanations for these results are as follows. First, two tests assess different aspects of swallowing. In VFSS, the patients swallow bolus of food or fluid in an upright position, whereas salivagram is used for detecting small amount of saliva in a supine position which occurs spontaneously or reflexively, and not by voluntary swallowing as required for VFSS [16]. In addition, both tests have high rates of false negative because aspiration is an intermittent phenomenon [10]. Therefore, a combination of VFSS and salivagram have an increased the efficacy of predicting aspiration pneumonia.

Several studies have compared VFSS and salivagram. Kim et al. [9] found that, among 110 children with swallowing difficulty, $43.6 \%$ (48/110) of children had positive findings in VFSS whereas $30.3 \%$ (33/110) of children had positive findings in salivagram. Jang et al. [14] prospectively studied 50 patients with aspiration pneumonia. Aspiration was observed in $42 \%(21 / 50)$ of patients by VFSS and in $34 \%(17 / 50)$ of patients by salivagram [14]. In this study, of the total 124 patients with chronic brain injury, $58.1 \%(72 / 124)$ of patients showed positive findings in VFSS and $55.6 \%(69 / 124)$ of patients had positive findings in salivagram. The sensitivity of VFSS was higher than that of salivagram, consistent with previous studies $[9,14]$. However, the percentage of positive findings in salivagram in this study (55.6\%) was higher than those in previous studies ( $30.3 \%$ and $34 \%$, respectively). Possible explanations for these results are as follows. First, a salivagram has a long monitoring time in the supine position which is physiologically similar to salivary swallowing. Second, a patient with chronic brain injury is more susceptible to salivary aspiration due to poor function with immobilization and swallowing dysfunction. Thus, a salivagram is more valuable for detecting salivary aspiration in patients with chronic brain injury than for other groups.

Several studies have evaluated salivary aspiration in patients with brain lesions using radionuclide salivagram. Kang et al. [17] have reported the utility of salivagram in 32 bedridden patients with brain lesion. Sex, cooperation for the response of MMSE and anterior drooling were associated with positive salivagram findings. Park et al. [11] performed salivagram and VFSS in 35 patients with brain injury. The MMSE score was found to be related to positive salivagram findings. The cut-off value of MMSE was 7 , suggesting that performing a salivagram could predict aspiration pneumonia in patients with cognitive impairment. Yu and Park [12] reported the clinical characteristics of salivagram findings in stroke patients with swallowing difficulty. The transfer sub-score of the MBI was significantly correlated with salivary aspiration in infratentorial stroke. In addition, MMSE score were a significantly correlated with salivary aspiration in supratentorial stroke. However, the findings of the current study were not consistent with those of previous studies as only swallowing functions among other clinical factors showed significant correlation with positive findings of salivagram in patients with chronic brain injury $[11,12,17]$. This discrepancy can be explained by disease the duration of the subjects. The present study focused on chronic patients in contrast to previous studies with a mean disease duration was 13.59 months (range, 3-64 months). In other words, they had a longer length of hospital stay and poorer functional state compared to that of patients from the other studies. In fact, only 11 patients (8\%) were on oral feeding while $113(92 \%)$ received tube 
feeding. One hundred patients (80\%) underwent tracheostomy. Mean MMSE (7.23 \pm 9.140$),$ MBI $(10.49 \pm 14.728)$ and FAC $(0.56 \pm 0.957)$ of this study were lower than those in previous studies $[11,12,17]$.

Swallowing reflex is a part of the swallowing phase under involuntary control. It occurs when the bolus is passed from the posterior tongue through the hypopharynx to the upper esophageal sphincter. It involves mechanoreceptors innervated by the internal branch of the superior laryngeal nerve. These mechanoreceptors are activated by swallowing. They are connected to central neurons that generate swallowing [18]. The activation of these receptors results in breathing stops secondary to vocal fold adduction, anterior-superior movement of the hyoid bone and larynx and epiglottic posterior movement to close the trachea. These movements are important defense mechanisms against aspiration. Several studies have performed kinematic analysis using VFSS. Seo et al. [19] demonstrated that persistent aspiration could have resulted from delayed triggering of the swallowing reflex. Choi et al. [20] found that higher epiglottic folding angles were significantly associated with recovery of swallowing function in subacute stroke patients. In the present study, triggering of pharyngeal swallowing, laryngeal elevation and epiglottic closure and nasal penetration were significantly associated with salivary aspiration on the salivagram. Loss of these airway protective mechanisms can increase the risk of salivary aspiration and pneumonia. Therefore, it would be advantageous to perform a radionuclide salivagram in a patient showing abnormal values for these parameters on VFSS.

The present study has several limitations. First, it was designed as a retrospective study which might have introduced selection and recall bias. Second, the sample size was relatively small and the patients were heterogeneous in the types of brain lesions. Therefore, the results of this study are not sufficient to make a general conclusion. Third, this study focused on patients with chronic brain injury. Therefore, the results of this study cannot be applied to patients with acute or subacute brain injury. Finally, all parameters of VFSS were quantified by first swallowing of a thin liquid $3 \mathrm{~mL}$ and foods with various viscosities were not used in this study. Therefore, further prospective studies with single large samples are recommended to identify the clinical predictors of aspiration pneumonia in patients with chronic brain injury.
This study showed a correlation between salivagram and VFSS findings. Salivary aspiration is not related the functional status but with swallowing function in chronic brain-injured patients. In conclusion, performing a salivagram in patients with less than 39 FDS in the pharyngeal phase might be helpful in preventing aspiration pneumonia in chronic brain-injured patients.

\section{CONFLICTS OF INTEREST}

No potential conflict of interest relevant to this article was reported.

\section{AUTHOR CONTRIBUTION}

Conceptualization: Shim GY, Oh JS. Methodology: Oh JS, Shim GY, Han SH. Formal analysis: Shim GY, Han SH. Project administration: Choi KY, Lee SM, Kim MW. Visualization: Shim GY. Writing - original draft: Shim GY, Oh JS. Writing - review and editing: Oh JS, Shim GY, Han SH. Approval of final manuscript: all authors.

\section{SUPPLEMENTARY MATERIALS}

Supplementary materials can be found via https://doi. org/10.5535/arm.20171.

\section{REFERENCES}

1. Martino R, Foley N, Bhogal S, Diamant N, Speechley M, Teasell R. Dysphagia after stroke: incidence, diagnosis, and pulmonary complications. Stroke 2005;36:2756-63.

2. Boesch RP, Daines C, Willging JP, Kaul A, Cohen AP, Wood RE, et al. Advances in the diagnosis and management of chronic pulmonary aspiration in children. Eur Respir J 2006;28:847-61.

3. Kang Y, Chun MH, Lee SJ. Evaluation of salivary aspiration in brain-injured patients with tracheostomy. Ann Rehabil Med 2013;37:96-102.

4. Kim SB, Lee SJ, Lee KW, Lee JH, Kim DW. Usefulness of early videofluoroscopic swallowing study in acute stroke patients with dysphagia. Ann Rehabil Med 2018;42:42-51.

5. Aviv JE, Sacco RL, Mohr JP, Thompson JL, Levin B, Sunshine S, et al. Laryngopharyngeal sensory test- 
ing with modified barium swallow as predictors of aspiration pneumonia after stroke. Laryngoscope 1997;107:1254-60.

6. Croghan JE, Burke EM, Caplan S, Denman S. Pilot study of 12-month outcomes of nursing home patients with aspiration on videofluoroscopy. Dysphagia 1994;9:141-6.

7. Smith Hammond CA, Goldstein LB, Horner RD, Ying J, Gray L, Gonzalez-Rothi L, et al. Predicting aspiration in patients with ischemic stroke: comparison of clinical signs and aerodynamic measures of voluntary cough. Chest 2009;135:769-77.

8. Yu KJ, Moon H, Park D. Different clinical predictors of aspiration pneumonia in dysphagic stroke patients related to stroke lesion: a STROBE-complaint retrospective study. Medicine (Baltimore) 2018;97:e13968.

9. Kim GE, Sung IY, Ko EJ, Choi KH, Kim JS. Comparison of videofluoroscopic swallowing study and radionuclide salivagram for aspiration pneumonia in children with swallowing difficulty. Ann Rehabil Med 2018;42:52-8.

10. Baikie G, South MJ, Reddihough DS, Cook DJ, Cameron DJ, Olinsky A, et al. Agreement of aspiration tests using barium videofluoroscopy, salivagram, and milk scan in children with cerebral palsy. Dev Med Child Neurol 2005;47:86-93.

11. Park D, Woo SB, Lee DH, Yu KJ, Cho JY, Kim JM, et al. The correlation between clinical characteristics and radionuclide salivagram findings in patients with brain lesions: a preliminary study. Ann Rehabil Med 2017;41:915-23.

12. Yu KJ, Park D. Clinical characteristics of dysphagic stroke patients with salivary aspiration: a STROBEcompliant retrospective study. Medicine (Baltimore) 2019;98:e14977.

13. Rosenbek JC, Robbins JA, Roecker EB, Coyle JL, Wood JL. A penetration-aspiration scale. Dysphagia 1996;11:93-8.

14. Jang DH, Choi KH, Kim DH, Lim CM, Kim JS. Comparison between the radionuclide salivagram and videofluoroscopic swallowing study methods for evaluating patients with aspiration pneumonia. Ann Nucl Med 2013;27:247-52.

15. Han TR, Paik NJ, Park JW. Quantifying swallowing function after stroke: a functional dysphagia scale based on videofluoroscopic studies. Arch Phys Med Rehabil 2001;82:677-82.

16. Lee HG, Oh SH, Lee YJ, Kim JS, Choi KH, Cho YA, et al. The use of radionuclide salivagram and videofluoroscopic swallow study in the evaluation of aspiration pneumonia in children. Pediatr Gastroenterol Hepatol Nutr 2012;15:160-5.

17. Kang IS, Kwon JG, Lee SU, Lee ZI, Park GY, Park HW. Detection of saliva aspiration using salivagram in bedridden patients with brain lesion. J Korean Acad Rehabil Med 2010;34:503-7.

18. Jafari S, Prince RA, Kim DY, Paydarfar D. Sensory regulation of swallowing and airway protection: a role for the internal superior laryngeal nerve in humans. J Physiol 2003;550(Pt 1):287-304.

19. Seo HG, Oh BM, Han TR. Longitudinal changes of the swallowing process in subacute stroke patients with aspiration. Dysphagia 2011;26:41-8.

20. Choi JS, Bang H, Lee GJ, Seo HG, Oh BM, Han TR. Epiglottic retroflexion is a key indicator of functional recovery of post-stroke dysphagia. Ann Rehabil Med 2020;44:1-10. 